Conclusion The Bu MR on day 3 above 4.973 and 25.06 were associated with worse EFS in children undergoing HSCT and received $\mathrm{Bu}$ in $\mathrm{QID}$ and $\mathrm{QD}$ dosing schedules, respectively.

Disclosure(s) Nothing to disclose

\section{P98 SEMI-QUANTIFICATION AND LOCALIZATION OF MEMBRANE TRANSPORTERS IN PAEDIATRIC KIDNEY TISSUE}

${ }^{1} \mathrm{M}$ van Borselen*, ${ }^{2} \mathrm{~B}$ van Groen, ${ }^{3}$ J Pertijs, ${ }^{3} \mathrm{M}$ Wilmer, ${ }^{4} \mathrm{~B}$ Smeets, ${ }^{5} \mathrm{R}$ Verdijk, ${ }^{2,3} \mathrm{~S}$ de Wildt. ${ }^{1}$ Radboud University, Nijmegen; ${ }^{2}$ Erasmus MC - Sophia Childrens Hospital, Rotterdam; ${ }^{3}$ Dept of Pharmacology and Toxicology, Radboudumc; ${ }^{4}$ Dept. of Pathology, Radboud Institute for Molecular Life Sciences, Nijmegen; ${ }^{5}$ Dept. of Pathology, Erasmus MC, Rotterdam, The Netherlands

\subsection{6/archdischild-2019-esdppp.136}

Background The kidney has a critical role in disposition, efficacy and toxicity of drugs and xenobiotics. Developmental changes of renal membrane transporters have the potential to explain population variability in paediatric pharmacokinetics and -dynamics of drugs but data are missing. We aimed to further delineate the expression of human renal tubular transporters multidrug resistance-associated protein (MRP) 4 and MRP2 and study localization in paediatric kidney samples.

Methods We planned to semi-quantify expression levels and to study the age-specific localization of the transporters MRP4 and MRP2 with immunohistochemistry on 44 human neonatal and paediatric kidney samples with age range of 24,00 - 40,00 weeks gestational age (GA) and 0,29 - 744 weeks post-natal age (PNA). The staining intensity was semi-quantitatively scored by two independent observers (MB and $\mathrm{BG}$ ).

Results MRP4 is found to be localized at the apical membrane of the renal proximal tubules at 27 weeks of GA $(n=3,1,29-4$ weeks PNA) and no age-related changes of expression levels were detected. In a premature neonate of 24 weeks GA $(n=1)$, no MRP4 was detected. The MRP2 staining did not meet the requirements to be scored and was rejected.

Conclusion MRP4 is expressed from at least 27 weeks GA onwards and does not show developmental changes. The localization was similar as in adults (Ritter et al., 2005). The half-life of the MRP4 substrate furosemide was found to be 6 to 20-fold longer in neonates than in adults (Pacifici, G.M., 2013). This could potentially be linked with the absence of MRP4 in a premature neonate with GA 24 weeks. However, these data should be confirmed as we only had 1 sample of \pm 24 weeks GA available. Moreover, our data help us in understanding altered disposition of transporter substrates in paediatrics.

\section{REFERENCES}

1. Pacifici, G. M. (2013). Clinical pharmacology of furosemide in neonates: a review. Pharmaceuticals;6(9):1094-1129.

2. Ritter CA, Jedlitschky G, Meyer zu Schwabedissen H, Grube M, Köck K \& \& Kroemer HK. (2005). Cellular export of drugs and signaling molecules by the ATP-binding cassette transporters MRP4 (ABCC4) and MRP5 (ABCC5). Drug metabolism reviews:37(1):253-278.

Disclosure(s) Nothing to disclose

\section{P99} CLINICAL VALIDATION OF PUBLISHED VANCOMYCIN POPULATION PK MODELS IN CRITICALLY ILL NEONATES

${ }^{1} A$ van der Veen*, ${ }^{2} R J$ Keizer, ${ }^{3} W$ de Boode, ${ }^{1} A$ Somers, ${ }^{4} R$ Brüggemann, ${ }^{4} R$ ter Heine, 1,5,6P De Cock. 'Department of Pharmacy, Ghent University Hospital, Ghent, Belgium; ${ }^{2}$ InsightRX, San Francisco, CA, USA; ${ }^{3}$ Department of Neonatal Intensive Care; ${ }^{4}$ Department of Pharmacy, Radboud University Medical Center, Nijmegen, The Netherlands; ${ }^{5}$ Heymans Institute of Pharmacology, Ghent University; ${ }^{6}$ Department of Pediatric Intensive Care, Ghent University Hospital, Ghent, Belgium

\subsection{6/archdischild-2019-esdppp.137}

Background Vancomycin is commonly used for treatment of severe Gram+ neonatal infections. Currently, even with the use of optimized dosing regimens and therapeutic drug monitoring (TDM), target attainment rates are abominable, leaving patients at risk for therapeutic failure and toxicity. Modelinformed precision dosing (MIPD) offers a large potential to improve therapy in the individual patient.

The aim of this study was to identify a suitable model for bedside MIPD by assessing the predictive performance of published population pharmacokinetic (popPK) models.

Methods A literature search was conducted to identify parametric popPK models. PK vancomycin data were retrospectively collected from NICU patients at the Radboud University Hospital, Nijmegen, The Netherlands. The model predictive performance was assessed by comparison of predictions to observations, calculation of bias (Mean Percentage Errors, MPE) and imprecision (Normalized Root Mean Squared Errors, NRMSE). Evaluations included both a priori (model covariate input) and a posteriori (model covariate and TDM concentration input) scenarios.

Results 265 TDM measurements from 65 neonates (median postmenstrual age:32 weeks [range:25-45 weeks]; median weight:1281g [range:597-5360g]; median serum creatinine: $0,48 \mathrm{mg} / \mathrm{dL}$ [range:0,15-1,28 $\mathrm{mg} / \mathrm{dL}$ ]) were used for model evaluation. Six popPK models were evaluated ${ }^{1-6}$. A posteriori predictions of all models were consistently more accurate and precise compared to the a priori (starting dose) predictions. PopPK models of Frymoyer et al. and Capparelli et al. consistently performed best through all evaluations in both the a priori and a posteriori scenario (MPE ranging from -18 to $6,4 \%$ in a priori scenario and 6,5 to $-3,8 \%$ in a posteriori scenario; NRMSE ranging from 34 to $40 \%$ in a priori scenario and 23 to $24 \%$ in a posteriori scenario).

Conclusion Large differences in predictive performance of popPK models were observed. Repeated therapeutic drug monitoring remains necessary to increase target attainment rate. Best performing models for bedside MIPD were identified in our patient population.

\section{REFERENCES}

1. Zhao W, Lopez E, Biran V, et al. (2013). Vancomycin continuous infusion in neonates: Dosing optimisation and therapeutic drug monitoring. Arch Dis Child;98 (6):449-453.

2. Capparelli EV, Lane JR, Romanowski GL, et al. (2001). The influences of renal function and maturation on vancomycin elimination in newborns and infants. $J$ Clin Pharmacol, 41:927-934.

3. De Cock RFW, Allegaert K, Brussee JM, et al. (2014). Simultaneous pharmacokinetic modeling of gentamicin, tobramycin and vancomycin clearance from neonates to adults: towards a semi-physiological function for maturation in glomerular filtration. Pharm Res;31(10):2642-2654.

4. Frymoyer A, Hersh AL, El-Komy MH, et al. (2014). Association between vancomycin trough concentration and area under the concentration-time curve in neonates. Antimicrob Agents Chemother, 58(11):6454-6461. 